\title{
Positive Air Pressure Device
}

National Cancer Institute

\section{Source}

National Cancer Institute. Positive Air Pressure Device. NCI Thesaurus. Code C70912.

A device for the delivery of forced air through a mask worn during sleeping hours, considered to be treatment for snoring and sleep apnea. 